\title{
A Hybrid Approach of Image Fusion using Modified DTCWT with High Boost Filter Technique
}

\author{
Vinay Sahu \\ Department of Computer Science Engineering \\ Shri SatyaSai College Of Engineering \\ Bhopal M.P.
}

\author{
Gagan Sharma \\ Department of Computer Science Engineering \\ Shri Satya Sai College Of Engineering \\ Bhopal M.P.
}

\begin{abstract}
To achieve improve quality of image two or more images are combined using a well known process, that process is called image fusion. The fused images provide us more information than the source images. Fusion process absolutely utilizes more entirely and surplus information. This paperproposes a hybrid approach using DTCWT with High boost filtering technique and the simulation of the proposed method is done in MATLAB2012a toolbox. The analysis of our method is performing among performance measuring parameter such MSE and PSNR in which analyze that our methodology gives improved results than the existing methods.
\end{abstract}

Keywords: HIS, PCA, Image fusion, Wavelet transform. MATLAB

\section{INTRODUCTION}

Image fusion is a process to blendmulti-sensorimages by using image processing techniques. Inexplicit image fusion means at integrating divergentand complementary data in order to augment theinformation perceptible in the images, as well as toenhance the dependability of the elucidation. Thisleads to more precise data and increased efficacy.Moreover, it is also acknowledged that the fused dataendow withvigorous operational performance such asincreased dependability, reduced vagueness, improvedassurance and better classification. Imagefusion is a method that endeavors at the integration ofincongruent and corresponding data to augment theinformation there in the source images as well asto augment the dependability of the elucidation. Thismethod leads to additionalprecise data explanationand efficacy [1].

The fusion process is nothing but a permutation ofprominent information in order to amalgamate an imagewith further information than individual illustration andamalgamated image is more appropriate for visualperception. It is used to denotea process by which manifold images or informationfrom manifold images is pooled. These imagesmay be achieved from unusual types of sensors.By means of the accessibility of the multisensor data innumerous fields, such as satellite imaging, remote sensing, medicalimaging or machine visualization, image fusion hasmaterialized as promising and significantinvestigationvicinity. On the other hand, image fusion is a practice ofunitingvarious input images of the identicalprospectinto a particular fused image, which safeguardswholecontent information and also preserving the imperativeattributes from all of the original images. The fusedimage must have additionalvaluable information contentcompared to the individual image.This method is classified into two categories: spatial domain and transform domain fusion. The images can be fused by different fusion techniques such as IHS (intensity hue saturation), PCA (principle component analysis) based fusion and transform based image fusion in which high pass filtering, pyramid and wavelet transform method is included. The HIS and PCA method belongs to spatial domain techniques while DWT and DCT comes into transform based fusion. The spatial domain techniques add up the distortion with the images. The transform based fusion is a powerful tool to fuse multisensory images. The performance of the image fusion techniques is analyzed using different parameter likewise PSNR (peak signal to noise ratio), entropy, MSE.

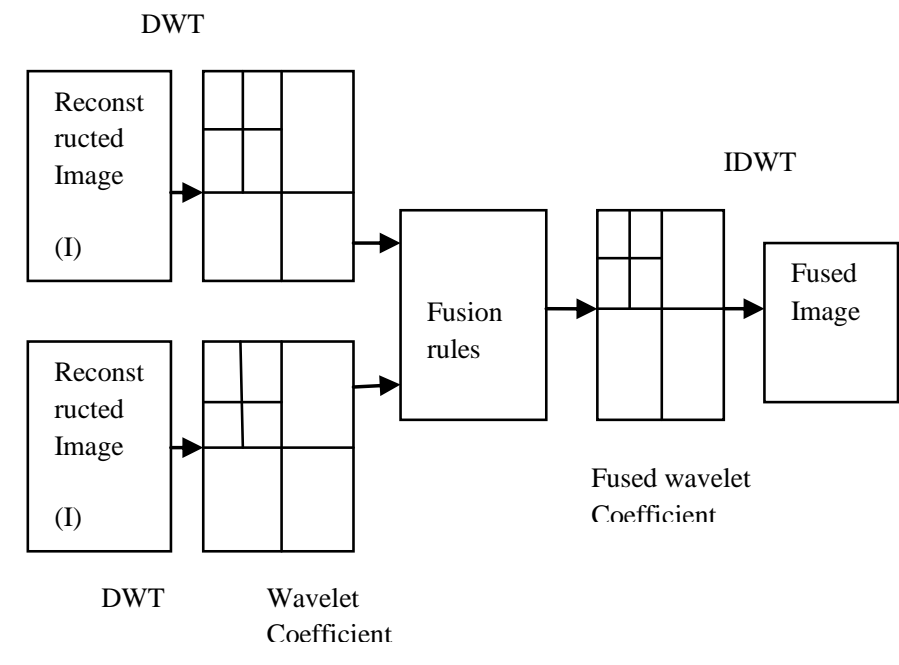

Fig. 1 Block diagram for image fusion process

The organization of the remaining section of the paper is as follows: Next section II describes the overview of the image fusion techniques. Section III presents the review of the previously work done in area of image fusion. In section IV describe our proposed methodology and section $\mathrm{V}$ shows the experimental analysis of the proposed method. Last section gives overall conclusion of the paper and its future scope.

\section{IMAGE FUSION TECHNIQUES}

The image fusion is basically classified in two categories namely: spatial domain and transform based fusion techniques. The spatial domain techniques includes following method like IHS, PCA and brovey method while transform fusion includes: high pass filtering, pyramid and wavelet transform. In this section some techniques of image fusion are describing below:

\subsection{Spatial domain method}

The spatial domain method [2], directly deals with the pixels of the input images. The pixel values are operate to achievemost wanted result. The example of spatial domain techniques is select maximum/ minimum fusion, HIS, PCA and brovey etc.

a) Select maximum/ minimum Method:

In this method [3], the consequential fused image is achieved by selecting the maximum intensity of subsequent pixels from both the input image. 


$$
F(i, j)=\sum_{i=0}^{m} \sum_{j=0}^{n} \operatorname{Max} A(i, j) B(i, j)
$$

$\mathrm{A}(\mathrm{i}, \mathrm{j}), \mathrm{B}(\mathrm{i}, \mathrm{j})$ are input images and $\mathrm{F}(\mathrm{i}, \mathrm{j})$ is fused image.

b) Select Minimum Method:

In this method [3], the resulting fused image is attained by selecting the smallest intensity of resulting pixels from both the input image.

$$
F(i, j)=\sum_{i=0}^{m} \sum_{j=0}^{n} \operatorname{Min} A(i, j) B(i, j)
$$

$A(i, j), B(i, j)$ are input images and $F(i, j)$ is fused image.

\section{c) IHS (Intensity Hue Saturation):}

The IHS method is one of the most regularly used fusion method for sharpening. It has become a standard process in image investigation for color enhancement, feature enhancement, enhancement of spatial resolution and the fusion of incongruent data sets [4]. Here the IHS space, spectral information is typically reflected on the hue and the saturation. As of the visual system, one can ends that the intensity modifies has modestconsequence on the spectral information and is uncomplicated to deal with. For the synthesis of the highresolution and multi-spectral remote sensing images, the principle is ensuring the spectral dataset and adding the additional information of high spatial resolution, consequently, the fusion is even more satisfactory for treatment in IHS space [5].

\section{d) Brovey Method:}

Brovey Transform is also known as the color normalization transform as it entails a red-green-blue (RGB) color transform method. It is statistical/numerical methods. Brovey Transform uses addition, division and multiplication for the fusion of three multispectral bands. The Brovey Transform was developed to visually enhance contrast in the small and high ends of an images histogram (i.e., to supply contrast in shadows, water, and high reflectance areas such as urban features). The Brovey transformation was developed to circumvent the disadvantages of the multiplicative method. The subsequent equation shows the mathematical algorithm for the Brovey method [9].

$$
F_{i}=\frac{M_{i}}{\sum_{i=1}^{N} M_{j}+P} \ldots \ldots \ldots \ldots
$$

Where Fi is the fused Band, Mi is the multispectral Band, P is the Panchromatic Band.

\section{e) Principal Component Analysis (PCA)}

PCA is a statistical tool which transforms a number of correlated variables into a number of uncorrelated variables. The PCA is used comprehensively in image compression and image classification. The PCA entails a mathematical process that transforms a number of correlated variables into a number of uncorrelated variables called principal components. It works out a squashed and optimal explanation of the data set. The first principal component accounts for as much of the variance in the data as probable and every succeeding component accounts for as much of the remaining variance as possible. Foremost principal component is taken to be alongside the direction with the maximum variance. The subsequent principal component is constrained to lie in the subspace perpendicular of the foremost. Surrounded by this subspace, this component points the direction of maximum variance. The third principal component is taken in the maximum variance direction in the subspace vertical to the initial two and so on. The PCA is also called as
Karhunen-Loève transform or the Hotelling transform. The PCA does not have a fixed set of basis vectors like FFT, DCT and wavelet etc. and its origin vectors depend on the data set [11].

\subsection{Transform Based Fusion}

The transform based fusion is an important tool to combine two or more image to obtain more supplementary information. In Transform domain fusion method image is initial transformed into frequency domain. Some of them transform based fusion is enlightened below:

\section{a) Discrete Wavelet Transform}

Wavelet theory is aconservatory of Fourier theory in many phases and it is introduced as an unconventional to the shorttime Fourier transform (STFT). In this, the signal is decomposed into sines and cosines however in wavelets the signal is anticipated on a set of wavelet functions. Fourier transform would supply good resolution in frequency domain and wavelet would make available good resolution in both time and frequency domains. Even though the wavelet theory was introduced as a mathematical tool in 1980s, it has been comprehensively used in image processing that supplies a multiresolution decomposition of an image in a bi-orthogonal basis and results in a non-redundant image demonstration. The basis is called wavelets and these are functions generated by transformation and dilation of mother wavelet. In Fourier analysis the signal is decomposed into sine waves of diverse frequencies. In wavelet analysis the signal is decomposedinto scaled (dilated or lengthened) and shifted (transformed) versions of the preferred mother wavelet or function. A waveletas its name involves is a small wave that cultivates and decays fundamentally in a limited time period. A wavelet to be a smallwave, it has to satisfy two basic properties [2]:

- Time integral must be zero

$$
\int_{-\infty}^{\infty} \varphi(t) d t=0 \ldots \ldots \ldots
$$

- Square of wavelet integrated over time is unity

$$
\int_{-\infty}^{\infty} \varphi^{2}(t) d t=0
$$

Wavelet transform of a 1-D signal $\mathrm{f}(\mathrm{x})$ onto a basis of wavelet functions is defined as:

$$
W_{a, b}(f(x))=\int_{-\infty}^{\infty} f(x) \varphi_{a, b}(x) d x=0
$$

\section{RELATED WORK}

In this section, describing some of the previously work done for fusing the multiple images to extract the more essential information by various researchers.

Kim et al. [8] initiated a new-fangled method based on the curvelet transform which represents edges better than wavelets. Since edges play anelementary role in image understanding, one good way to develop spatial resolution is to augment the edges. Curvelet-based image fusion method presentsmore affluent information in the spatial and spectral domains simultaneously. They performed IKONOS image fusion. This novel method has accomplished an optimum fusion result.

Rana et al. [9] explored unusual medical image fusion methods and their comparison to discover out which fusion method gives better results based on the performance parameters. Here 
medical images of Magnetic Resonance Imaging (MRI) and Computed Tomography (CT) images are fused to form fresh image. This novel fused image progresses the information content for diagnosis. Fusing MRI and CT images present more information to doctors and clinical treatment planning system. MRI presentsimproved information on soft tissues whereas CT presentsimproved information on denser tissues. Fusing these two images bestows more information than single input image. In this, wavelet transform, Principle Component Analysis (PCA) and Fuzzy Logic techniques are utilized for fusing these two images and outcomes are compared.

Singh et al. [10] proposed a fresh detail-enhancing revelation fusion method using non-linear translation-variant filter (NTF). Due tothe captured Standard Dynamic Range (SDR) images under unusual exposure settings, primary the excellent details are extracted basedon guided filter. Subsequently, the base layers (i.e., images obtained from NTF) transversely all input images are fused using multi-resolutionpyramid. Exposure, dissimilarity, and saturation measures are considered to engender a mask that guides the fusion process of the baselayers. In conclusion, the fused base layer is combined with the digger out fine details to achieve detail-enhanced fused image. The purpose is tosafeguard details in both tremendously dark and extremely bright regions without High Dynamic Range Image (HDRI) representation andtone mapping step. Furthermore, they had demonstrated that the proposed method is also appropriate for the multifocus image fusionexclusive of introducing artifacts.

Mini et al. [11] proposed an augmentation technique which exploits Stationary Wavelet Transform (SWT), modulus maxima and high boost filtering. The image is decomposed using SWT and its modulus maximum is indomitable. A division of the high pass filtered image achieved as the result of SWT decomposition and modulus maxima is supplementary to original image. The system is evaluated visually and dispassionately using measures like dissimilarity, PSNR etc. The performance measures are appraised for dissimilarclass of images and found to be appropriate to all categories of mammographic images.

Tsagariset al. [12] proposed a method forhyper-spectral image fusion based on principalcomponents transformation (PCT). The foremostprincipal component of every subgroup is used forimage visualization. The number of bands in allsub-groups depends on the application. On the other hand, intheir work, three sub-groups were used for RGBrepresentation. One of the sub-groups employedmatched filtering based on the spectralcharacteristics. The excellence of the obtained RGBimages were quantitatively estimated usingparameters like, correlation coefficient, entropy, and the maximum energyminimum correlationindex. The performance of their technique incategorization has been tested using the K-meansalgorithm.

\section{PROPOSED WORK}

In this section we are describing our work to extract the essential features by fusing the multiple images from image dataset. In our work DTCWT and Filtering techniques is used to fuse the images.

\subsection{DT-CWT (Dual Tree Complex Wavelet Transform)}

In the dual tree complex wavelet transform method, [13] the fusion is performed using masquerades to dig out information from decomposed structure of DT-CWT [13]. The DT-CWT arrangementengages both the real and complex coefficients.
This complex transform employs two detach DWT decompositions. It is divided into two detach trees, tree A and tree $\mathrm{B}$ as revealed in figure 2 below.

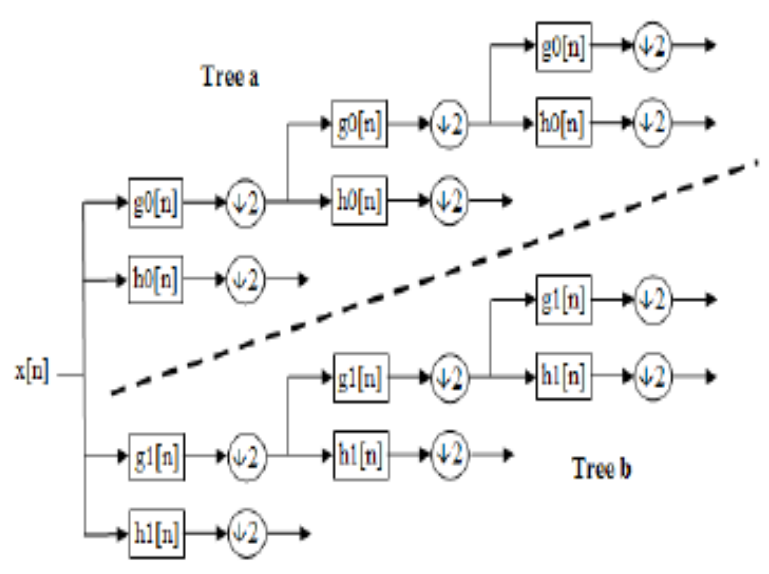

Fig.2 Structure of DT-CWT

The fusion progressionabsorbs the formation of the fusedpyramid using DT-CWT coefficients which are achieved from the decomposed pyramids of the input source images. The fused image is fashioned by applying inverse DT-CWT transform. DT-CWT preserves the restrained texture regions of brain in MRI images. Ringing outcomes are reduced and edge details are also retained more obviously. This DT-CWT is more beneficial than the DWT method as it is directional sensitivity and shift variant in behavior.

\subsection{Fusion Rule:}

Entropy is the determination of information content in the image. A high value of entropy represents moreinformation content and vice versa. Consequently this statistical determination could be used in making a decisionto choose the fusion coefficient [14].

$$
H(S)=-\sum P(X) \log p(X)
$$

Entropy is premeditated on the low frequency components of the input images surrounded by a 3-by-3window and either having higher values of entropy were selected or the fusion coefficientsamong the low frequency components.

Subsequently the coefficient is selected as the fuse coefficient when the region energy of it is better shownas formula

$$
F_{p}^{k}(i, j)=\left\{\begin{array}{l}
A_{p}^{k}(i, j), \text { if } A_{p}^{k m}(i, j)>B_{p}^{k m}(i, j) \\
B_{p}^{k}(i, j) \quad \text { Otherwise }
\end{array}\right.
$$

In conclusion the fused image is reconstructed using the fused coefficients $F_{p}^{k}(i, j)$ using the inverseNSCT transform.

4.3High pass filters: A high pass filter is mostly used for sharpening purpose. When an image is sharpened and contrast is superior between bordering areas with little variation in brightness or low eminence information.

High pass $=f(x, y)-$ low pass

4.3.1 High-boost Filtering: A high-boost filter is also identified as a high-frequency prominence filter. A high-boost filter is used to preserve several of the low-frequency apparatus to abet in the elucidation of an image. In high-boost filtering 
input image $\mathrm{h}(\mathrm{m}, \mathrm{n})$ is multiplied by an amplification factor $\mathrm{A}$ prior to subtracting the low-pass image. Accordingly, the highboost filter expression is:

$$
\text { High Boost }=A * f(m, n)-\text { low pass }
$$

Adding and subtracting 1 with gain factor, then High boost $=(A-1) * f(x, y)+f(x, y)-$ low pass So

$$
\begin{array}{r}
\text { High Boost }=(A-1) * f(x, y) \\
+ \text { High pass } .
\end{array}
$$

\subsection{Proposed Algorithm}

1. Select Image A from database

2. Select Image B from database

3. Apply DTCWT and decomposed images into sub-bands(LL, LH, HL, HH)

4. Wavelet coefficient into A and B

5. Apply transform function on A, B

6. Apply high pass filter into decomposed layers of $\mathrm{A}$ and $\mathrm{B}$

7. Apply Gaussian filter to removes extra noise from decomposed layers

8. Then apply high boost filter to improve intensity of each decomposed layers

9. Apply fusion rule:

$$
F_{p}^{k}(i, j)\left\{\begin{array}{l}
A_{p}^{k}(i, j), \text { if } A_{p}^{k m}(i, j)>B_{p}^{k m}(i, j) \\
B_{p}^{k}(i, j) \quad \text { Otherwise }
\end{array}\right.
$$

10. Then fused all improved wavelet coefficients

11. Apply INV-DTCWT function

12. Now Compute MSE is as follows:

$$
\text { MSE }=\frac{1}{m n} \sum_{i=1}^{m} \sum_{j=1}^{n}\left(A_{i j}-B_{i j}\right)^{2}
$$

13. And compute PSNR as follows:

$$
\text { PSNR }=10 \times \log _{10}\left(\frac{\text { peak }^{2}}{\text { MSE }}\right)
$$

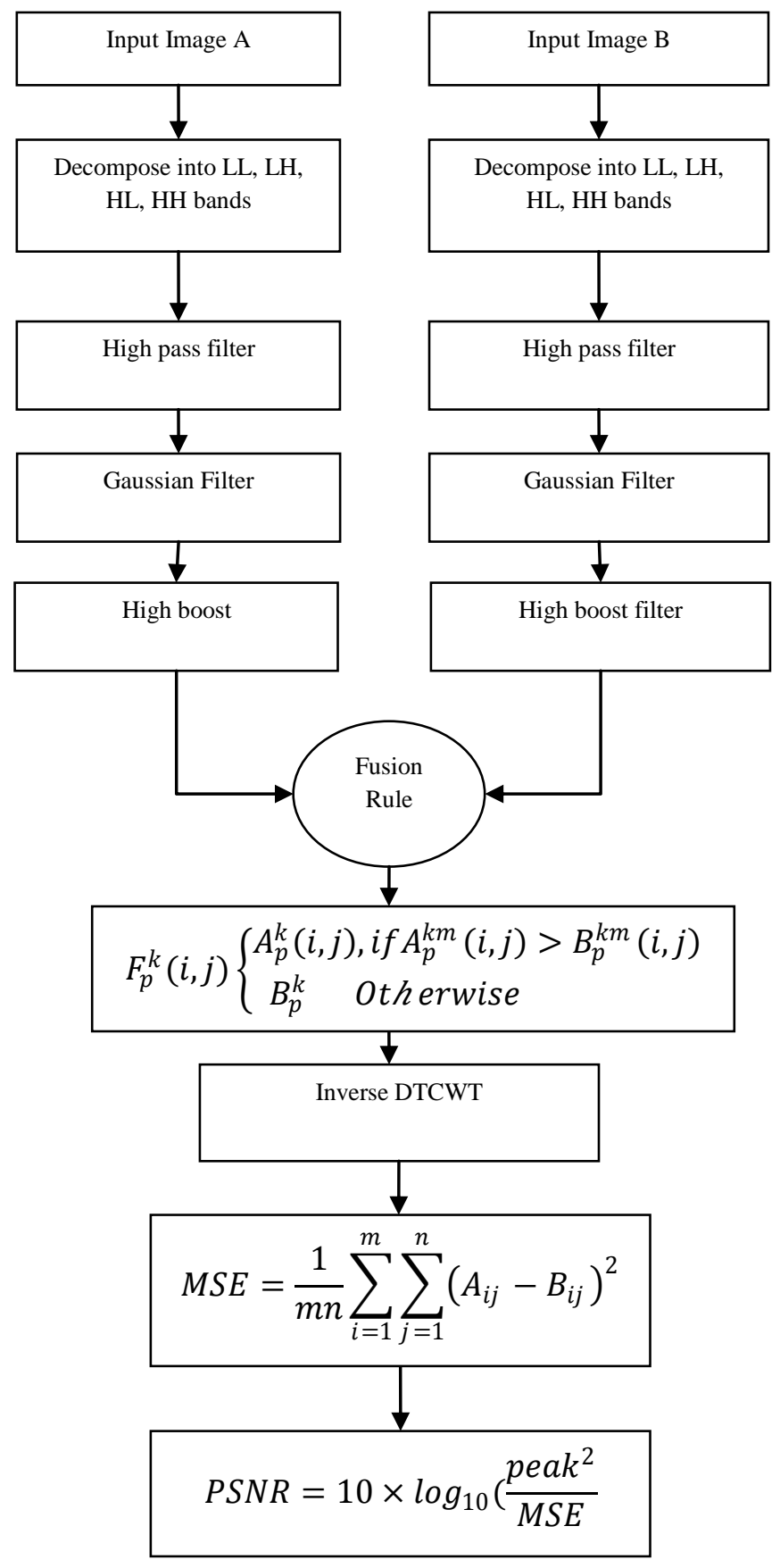

Fig. 3 Block diagram for proposed methodology

Here block structure of proposed methodology shows that initially, we have taken the input of two images from the dataset and then decomposes it in the form LL, LH, HL, HH sub bands by applying DT-CWT technique. Later then, apply high pass filter into decomposed layer of input image A and B. Again introduce Gaussian filter to sharp or eliminating the extra noise from the decomposed layer. After applying Gaussian filter apply High Boost filter to improve the intensity of each decomposed layers. Then apply fusion rule to blend the decomposed layer and finally apply inverse DT-CWT to get the fused image with more information. 


\section{EXPERIMENTAL RESULTS}

In this section,we show the implementation and analysis of our proposed work and the implementation is done using the $\mathrm{C}++$ programming language. The simulation of our work is done using the well known MATLAB2012a [15]. The analysis is done among different image dataset and comparison done by PSNR and MSE. From this analysis, observe that our proposed methodology give better results than the DWT and DTCWT image fusion technique. For comparing the methods, they have tested four repudiated images set namely: Saras, clock, balloon and leopard.And here Fig. 4, fig. 5 show the main GUI environment of implemented system which contains the all compared method and illustrate the result after successful completion, here they observed that the MSE is less at most time and PSNR is higher of proposed method as compared with DWT and DTCWT.

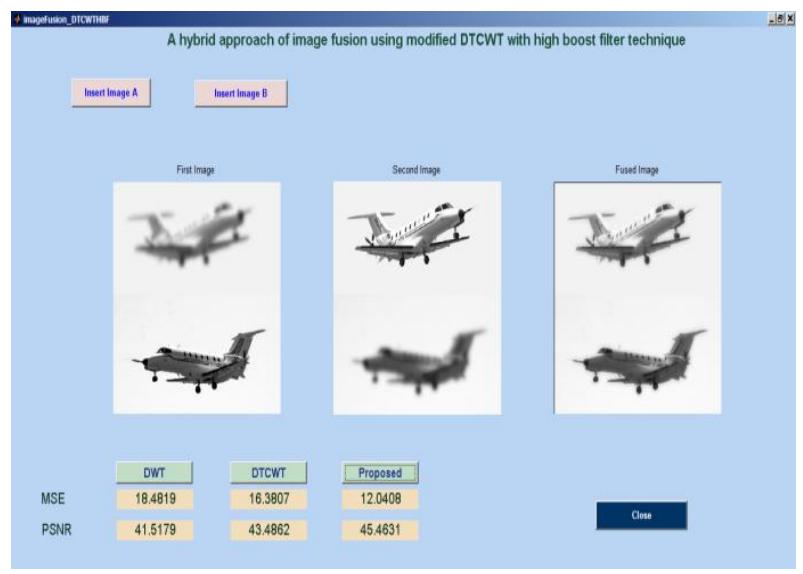

Fig. 4 GUI environment for saras.jpg

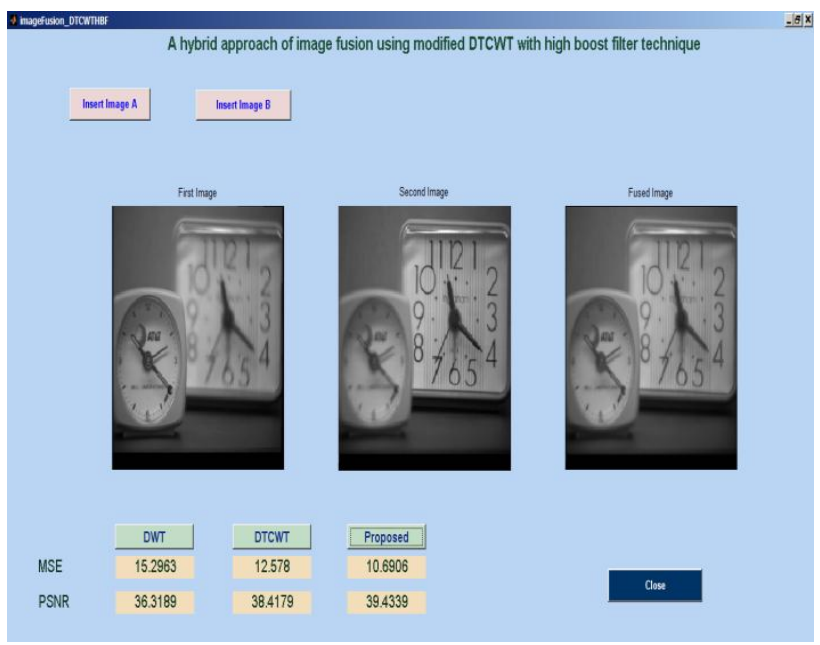

Fig. 5 Clock fused image dataset

Here table 1 and table 2 shows the comparison of the proposed method among DWT and DTCWT in which observe that simulation result is much better for MSE and PSNR performance parameter and it is illustrated through chart.

Table 1 MSE comparison of proposed method

\begin{tabular}{|l|c|c|c|}
\hline \multicolumn{4}{|c|}{ MSE comparison } \\
\hline Method/image & DWT & DTCWT & Proposed \\
\hline saras.jpg & 18.4819 & 16.3807 & 12.0408 \\
\hline clock.png & 15.2963 & 12.578 & 10.6906 \\
\hline
\end{tabular}

\begin{tabular}{|l|c|c|c|}
\hline baloon.png & 9.00496 & 7.16232 & 11.8188 \\
\hline lapord.png & 18.133 & 14.7277 & 12.8613 \\
\hline
\end{tabular}

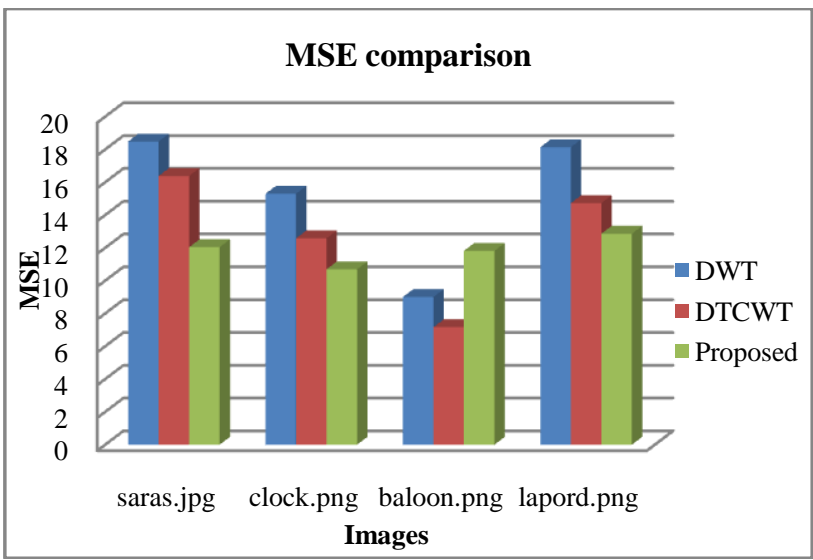

Fig. 6 MSE comparison of DWT, DTCWT and proposed method

Table 2 PSNR comparison of all methods

\begin{tabular}{|l|c|c|c|}
\hline \multicolumn{4}{|c|}{ PSNR comparison } \\
\hline Method/image & DWT & DTCWT & Proposed \\
\hline saras.jpg & 41.5179 & 43.4862 & 45.4631 \\
\hline Clock.png & 36.3189 & 38.4179 & 39.4339 \\
\hline baloon.png & 39.3088 & 41.6777 & 39.7112 \\
\hline leopard.png & 36.2689 & 38.4216 & 39.309 \\
\hline
\end{tabular}

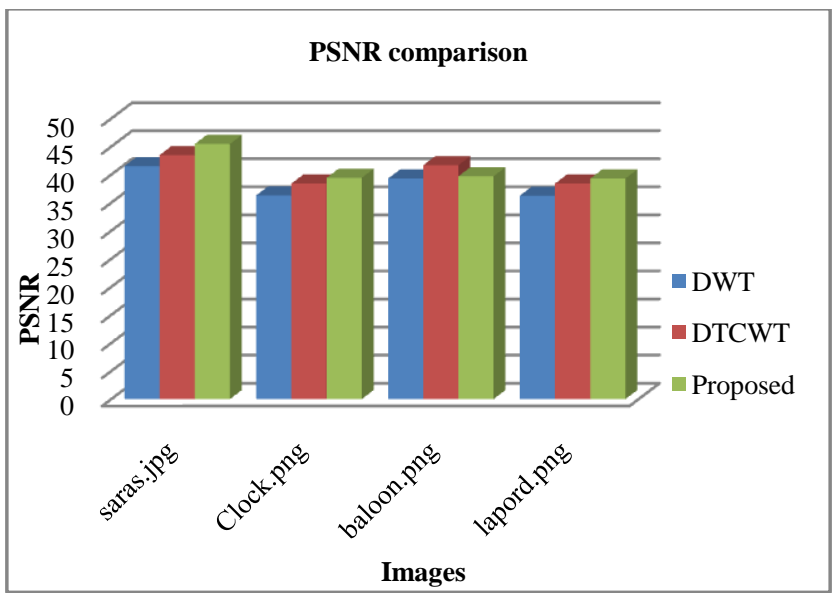

Fig. 6 PSNR comparison of DWT, DTCWT and proposed method

\section{CONCLUSION}

From the single image it is not possible to extract the essential or more information so to obtain more information the multiple images are fused together. Various techniques have been developed to fuse the images. In this paper, hybrid approach using DTCWT with high boost filtering is proposes. The analysis of this work is done using MSE and PSNR measuring parameter and the simulation results of proposed method gives better results than the existing methods. In future work, analyze this methodology among more performance measuring parameter except PSNR and MSE. 


\section{REFERENCES}

[1] S. Anbumozhi, P.S. Manoharan, "Performance Analysis of Fusion Based Brain Image Classification Using Minimum Distance classifier", Journal of Theoretical and Applied Information Technology 20 April 2014. Vol. 62 No.2, ISSN: 1992-8645.

[2] C. Morris \& R. S. Rajesh, "Survey of Spatial Domain Image fusion Techniques", International Journal of Advanced Research in Computer Science Engineering and Information Technology Volume: 2 Issue: 3 08-Apr-2014, ISSN NO: 2321-3337.

[3] Kusum Rani, Reecha Sharma, "Study of Different Image fusion Algorithm", International Journal of Emerging Technology and Advanced Engineering. Website: www.ijetae.com (ISSN 2250-2459, ISO 9001:2008 Certified Journal, Volume 3, Issue 5, May 2013).

[4] Pohl C. and Van Genderen J. L., "Multi-sensor Image Fusion In Remote Sensing: Concepts, Methods And Applications", International Journal Of Remote Sensing 1998, Vol. 19, No.5, pp. 823-854.

[5] Hui Y. X. and Cheng J. L., 2008. "Fusion Algorithm for Remote Sensing Images Based on Non sub sampled Contour-let Transform", ACTA AUTOMATICA SINICA, Vol. 34, No. 3.pp. 274- 281

[6] Sunil Kumar Panjeta and Deepak Sharma "A Survey on Image fusion Techniques used to Improve Image Quality", International Journal of Applied Engineering Research, ISSN 0973-4562 Vol.7 No.11, 2012.

[7] V.P.S. Naidu and J.R. Raol, "Pixel-level Image Fusion using Wavelets and Principal Component Analysis". Defense Science Journal, Vol. 58, No. 3, May 2008, pp. 338-352 Ó 2008, DESIDOC.
[8] MyungjinChoi , Rae Young Kim, Moon-Gyu Kim, "The Curvelet Transform For Image Fusion".

[9] Rana, A. and S. Arora, "Comparative analysis of medical image fusion". International Journal Computer Application 2013, 73:10-13. DOI: 10.5120/12768-9371.

[10] Harbinder Singh Vinay Kumar and Sunil Bhooshan, "A Novel Approach for Detail-Enhanced Exposure Fusion Using Guided Filter", Hindawi Publishing Corporation the Scientific World Journal Volume 2014, Article ID 659217.

[11] Arya Devi P. S. and M. G. Mini, "Mammographic Image Enhancement Based on SWT and High Boost Filtering", International Journal of Computer Theory and Engineering, Vol. 7, No. 5, October 2015

[12] V. Tsagaris, V. Anastassopoulos, and G. Lampropoulos, "Fusion of hyperspectral data using segmented PCT for enhanced color representation", IEEE Trans. Geosci. Remote Sens., Vol. 43, No. 10, 2005, pp. 2365-2375.

[13] A.Harika, "Image Fusion Based On Dtcwt\&PcaIn Presence Of Noise", International Journal of Advanced Research in Computer and Communication Engineering Vol. 3, Issue 11, November 2014, ISSN (Online): 2278-1021.

[14] Manu V T and Philomina Simon "A Novel Statistical Fusion Rule For Image Fusion And Its Comparison In Non Sub-sampled Contourlet Transform Domain And Wavelet Domain", International Journal of Multimedia \& Its Applications (IJMA) Vol.4, No.2, April 2012.

[15] http://in.mathworks.com/matlabcentral/answers/40897links-to-matlab-documentation-in-published-html-pages. 